\title{
A Healthy Community through Health System Partnerships: The Approach of Markham Stouffville Hospital
}

\author{
To \\ COM MEN TARY \\ James R. MacLean, MD \\ President and CEO, Markham Stouffville Hospital \\ Lorne Zon, BA, MES \\ Vice President, Markham Stouffville Hospital \\ $\propto$
}

The STRUCture of THE CANADian HEALTHCARE SYSTEM, particularly in Ontario, has remained remarkably stable over the past 25 years. No other private sector industry employing hundreds of thousands of people, spending tens of billions of dollars annually and serving millions of consumers every day has survived for 30 years without the need to reinvent itself in quite fundamental ways. How then has the healthcare sector in Canada avoided the pressure to "reinvent itself?"

The answer, of course, is that changes have occurred in many elements of healthcare. Clinically, treatment modalities have made tremendous strides. Training of professionals is very different than it was three decades ago. Funding has shifted in subtle, but important ways. But, in Canada, as in healthcare systems around the world, the quest for the almost mythical organization for the delivery of health services that can provide accessible, efficient and cost-effective care remains illusive. For the most part, the basic delivery structures remain much the same - in uncoordinated silos. 
With all the changes that have occurred, one critical element remains fairly much intact - the patient's experience. As boomers reach middle age and take on the role of caregivers to their parents, the frustration level with the current system increases. Service gaps and lack of coordination and information challenge the families to put together the services they need.

In the mid-1990s, the Canadian ongoing search for the "right" system focused on regionalization. In all provinces except Ontario, some level of regionalization has occurred. The theory behind the move to regional authorities is simple and powerful:

1. Decisions about resource allocation are better closer to the populations being served.

2. Fragmentation can be reduced if the number of corporate entities is reduced.

Together, these conditions can provide the foundation for achieving a health system:

- that is rooted in population health;

- that is driven by a common vision; with a unified management to operationalize the vision; and

- that has an accountability framework to the people (via the regional board).

However, the theory, as yet, has not been proven in the field. The results of regionalization, though evaluation is sparse, appear to be less than spectacular. Certainly some positive benefits have been achieved. However, the stresses and strains on healthcare services have remained largely unchanged.

\section{The Ontario Experience}

In Ontario, a different path was taken. In the mid-1990s, three paradigms were beginning to emerge:

- The integrated health system (IHS), as an alternative to regionalization, was gaining considerable momentum as the model of choice among providers.

- Hospital restructuring as a precursor to total system reform was in full swing in almost every Ontario community.

- The Ministry of Health was contemplating a move to a decentralized regional office structure as an alternative to regional authorities.

One of the three paths came to a dead end. The negotiated agreement between the government and the Ontario Medical Association (OMA) included certain conditions that were perceived, by champions of the IHS movement, as being the final chime of the death knell for integration. Restrictions on innovative use of the physicians' "fee-for-service pot" meant that introducing alternative payment plans (for example, capitated payments) became highly unlikely. Since rostering and capitation were considered the backbone of an IHS (HMRU 1996), the agreement was like a pail of cold water being thrown on those pressing for new, population-health-based initiatives.

Following the signing of the OMA agreement, the Ministry of Health quietly began disbanding the policy group that was researching integrated health systems. Community Care Access Centres were established as a new stand-alone entity. Hospital restructuring and decentralization continued but the fundamental relationships between health providers in the healthcare delivery system remained impervious to change. 


\section{Has the IHS Ship Come In or Sailed into the Sunset?}

Leatt, Pink and Guerriere take Shortell's notion of an "organized delivery system" and break it down into several fundamental elements. Components include:

- focusing on community health needs and the capacity to meet these needs;

- coordination of a continuum of care;

- information linkages; and

- using incentives to align governance, management, physicians and other providers.

Again referring to Shortell, the authors note that today's delivery is more akin to multiple, independent structures built around the provider rather than the consumer.

Analyzing the gap and the possibilities for moving forward, the authors provide some insightful findings from their review of international experience. Of significant importance are the six interrelated strategies for moving ahead:

- Focus on the individual.

- Start with primary healthcare.

- Share information/exploit technology.

- Create virtual coordination networks at the local level.

- Develop practical needs-based funding models.

- Implement mechanisms to monitor and evaluate.

Ontario's providers have promoted a top-down approach to system reform.

They have advocated for legislation and policy to create the framework, incentives and funding models to allow an IHS to develop and flourish (TDHC 1996). Given the lack of provincial action to date, is there an alternative? Can local initiatives be implemented without government support or intervention? In the following paragraphs the efforts of Markham Stouffville Hospital (MSH) will be examined against the six points that are contained in "Towards a

Canadian Model of Integrated Healthcare.” MSH's plans and actions will be used to determine the viability of a bottom-up approach to building an integrated health system.

\section{Markham Stouffville Hospital's Approach}

MSH has supported and promoted the IHS concept for several years. The application of the approach to a local health system was first outlined in a 1995 acute care study carried out by the York Region District Health Council (1995). It was further refined in a subsequent report entitled "Southeast York Region Integrated Health System: Creating Canada's Healthiest Community" (Avrich et al. 1997). Like most of the initiatives of that time, its implementation model required Ministry agreement, support and participation. To date, none of these conditions has been achieved.

In its review of York Region, the Health Services Restructuring Commission (HSRC 1997) supported the move to integrated health system development in York Region as recommended by the DHC and supported by the hospitals. In 1999, MSH decided that a locally led initiative was the only option for immediate action. The hospital believes that change is possible, and that by taking a visionary, perhaps risky, leadership role in creating a virtual IHS, the benefits will encourage government and other healthcare providers to move forward. 
The MSH vision, approved in January 2000, will provide the guidepost for action:

"Achieving a healthy community through health system partnerships."

The vision builds on the previous work of the hospital and its partners, but emphasizes the foundation of partnership rather than new corporate structures. An examination of the current and proposed strategies against the six lessons outlined above is provided here.

\section{Focus on the Individual}

York Region, where Markham Stouffville Hospital is located, is experiencing one of the fastest growth rates in Canadareceiving 66 new residents each day. As well, the historically homogeneous population is becoming quite multicultural. As in all of Ontario's communities, the population is also aging. To better understand and serve individuals and families requiring service, the hospital has begun to work closely with the Simcoe-York District Health Council to utilize its recently completed comprehensive community profiles. This work is complemented by the preparation of stakeholder dossiers that will highlight the needs, wants and expectations of key groups that utilize and support the hospital.

The rapidly changing demographics of the population have challenged Markham Stouffville Hospital and other local health providers' abilities to provide needed services. Partnerships provide a real and viable means of addressing these challenges. For example, to better serve the Chinese community, the hospital is developing a strategic partnership with the Yee Hong Centre for Geriatric Care. This innovative, long-term care centre can provide the hospital with improved capacity to respond to individual cultural needs. In return, the hospital can provide necessary secondary services to the centre's residents and community clients. A further strategy is aimed at helping individuals who live in the area with health education and health promotion information. In addition to the many ambulatory clinics that provide individuals with counselling, the hospital is redesigning its website to directly provide or link individuals seeking information to reliable and up-to-date sources.

\section{Start with primary healthcare}

Rosser and Kasperski (1999) reiterate the position of the Ontario College of Family Physicians that family practice is based on four principles:

1. The patient-physician relationship.

2. The family physician as a skilled clinician.

3. The family physician as a resource to a defined practice population.

4. Family medicine as a communitybased discipline.

They go on to note that "current healthcare systems do not provide incentives or any support for family physicians to practise according to these four principles." If, as often noted, the development of an IHS is rooted in primary care, and if primary care requires appropriate incentives to bring change about, then what can be done without government intervention? MSH believes that some incentives are possible within existing legislation, although fundamental change will require a more enabling OMA/ Government agreement and changes 
to fiscal policy. The family physicians in Ontario have supported changes to fee for service for several years (OCFP 1995).

The mutual benefits derived from closer linkages between a hospital and its primary care physicians have been studied by a number of groups. The Toronto District Health Council (TDHC) held an invitational conference for family physicians in March 1999. The conference proceedings (TDHC 1999) documented a significant frustration by doctors regarding the number of hours spent each week trying to find community services for patients. These efforts were unpaid, undervalued and took away from the physicians' capacity for one-on-one care. Providing what is often termed case management is a primary care responsibility. But is this service best undertaken by a family physician? The Ontario College of Family Physicians (OCFP 1999) points out that solo practice or small group practice cannot provide the infrastructure to undertake these responsibilities.

MSH has been working with two physician practices (comprising 17 family physicians) on integrating health records and providing technology links. This innovative approach will be described in more detail below. Several other types of hospital/physician partnerships will also be undertaken to assist in establishing a primary care base for integrated health delivery.

In the absence of regional or provincial structures, a local hospital may be the only provider with a sufficient resource base to provide the resources needed for case management to support primary care physicians using other types of health professionals. MSH is looking at several strategies, in addition to technology, to support local groups of family physicians. The hospital is considering ways to provide financial incentives to physicians to contribute to providing non-fee-for-service benefits to clients and by involving them in a virtual, multidisciplinary team including nurse practitioners, social workers, pharmacists and other healthcare professionals. The hospital is also researching the potential of offering its sophisticated management support to physician groups to improve their operational efficiency.

\section{Share information and exploit technology}

In 1996 the Ontario Ministry of Health held a series of focus groups on integrated healthcare systems. "The overwhelming advice in most of the focus groups was that the design and funding of affordable, accessible and appropriate information systems is pivotal to IHS implementation" (Quigley 1996). MSH has invested considerable efforts and its own funding in developing technological links to its physicians inside and outside the hospital. Two physician practices have entered into a pilot project with the hospital to link patient records and improve information flow. If successful, this model can be expanded to other physician groups, both primary and specialist. The current investment by MSH with its physician partners will pay considerable dividends in reduced duplication of tests and better and faster communication between specialists, Community Care Access Centres and the family physician. Greater coordination and continuity of care should improve health outcomes and lead to higher levels of patient satisfaction. 
Create virtual coordination networks at the local level

Goldsmith (1998), a leading healthcare futurist, notes that a virtual IHS can produce the same if not greater benefits than the single corporation model. Virtual IHSs build on the strengths of each partner. Markham Stouffville Hospital is advocating a multitiered strategy to build a virtual integrated health system for its community. Central to the strategy is a local, vertically integrated network of agencies that will address the primary and secondary care needs of the community. As a starting point in building the network, the emphasis for the next year will be on long-term care and rehabilitation (in addition to primary care reform discussed above).

In the area of long-term care, the hospital has initiated discussions with local long-term care facilities and the York Region Community Care Access Centre. The hospital will be working with the agencies to design and implement a specialized geriatric service to address current and emerging population health needs and build on the strengths and capacities of the community providers. As several of the agencies are planning major capital redevelopment, joint clinical space and program planning will be encouraged.

Rehabilitation is another area for action. A local physiotherapist coalition and MSH have been developing a rehabilitation network agreement. The network will provide one-stop information and referral service to residents seeking care, joint needs-based planning and program development, and standard setting and monitoring. This private/ not-for-profit arrangement is a potential model for other service developments.
The second tier of the strategy is directed towards horizontal integration. Markham Stouffville Hospital is a medium-size, suburban community hospital providing a comprehensive range of secondary care. The geographic location of Markham Stouffville Hospital results in travel patterns that lead southward from the Uxbridge site of the Lakeridge Hospital System to Markham Stouffville Hospital and for more regional/specialized care to the Rouge Valley Health System (a large, multisite community hospital). Considerable synergy exists to develop a comprehensive range of hospital-based primary, secondary and (non-teaching) tertiary-based care through horizontally integrated partnerships among the three hospitals. As one of the three hospitals in York Region, MSH has also developed joint programs with the other two hospitals in the region. A shared MRI service is one development that has been a successful collaborative undertaking.

The third tier in our integration strategy links all the local hospitals serving northeast Toronto, southeast York Region and Durham Region with a full-service teaching centre for educational and highly specialized care. This academic network includes the Sunnybrook and Women's College Health Science Centre (three sites), Markham Stouffville Hospital, York Central Hospital, North York General Hospital (two sites), Rouge Valley Health System (two sites) and Lakeridge Health System (five sites). Together, this horizontal network serves over 1.5 million people. The network can provide a wide variety of educational and training opportunities for the physicians and hospital staff of all participating 
member institutions. A second specialtybased academic and program partnership is being discussed with the St. John's Rehabilitation Hospital. St. John's has been designated by the Health Services Restructuring Commission as a regional (physical) rehabilitation referral centre.

\section{Develop practical needs-based funding models}

Of the six lessons learned, this is beyond the scope of our hospital alone to embrace. What can be done is to begin to develop with the Simcoe York DHC and others a needs-based planning and monitoring capacity. The information collected will inform Ministry action when changes in funding are required due to developments related to care needs or population growth.

\section{Implement mechanisms to monitor and evaluate}

Accountability frameworks, report cards and integrated information systems are necessary and expected in today's healthcare environment. MSH is taking an "educated" risk in moving ahead with its strategies without explicit Ministry commitment and support. It is essential that the initiatives are carefully monitored and evaluated by a third party so that the successes and failures can be analyzed and understood. MSH will work with the Simcoe York District Health Council and evaluation experts to design and collect ongoing information so that an appropriate evaluation of the projects can be done and lessons can be disseminated to the Ministry of Health and Long-Term Care as well as others contemplating similar projects.

\section{Where Do We Go from Here?}

As MSH embarks on this path to local integration one question remains unanswered: Why now? The experience to date with changes is not particularly encouraging. Several factors are coming together to lend support to our efforts:

- The rising curve of the aging population and the much more modest rise in health expenditures means changes must take place and reasonably soon.

- Information technologies have become more sophisticated, and linkages of various platforms to establish an integrated clinical record are now available.

- Public expectations continue to grow.

- In Ontario, a driving force will be balanced budget legislation. If better delivery methods are not found, financial pressures will outstrip the government's ability to raise revenues.

- Finally, provincial and federal policy musings are increasingly supportive of reform.

Leatt, Pink and Guerriere state that there is no one answer to building successful integrated health systems. Rather, they conclude that "a variety of strategies must be tried in different communities." The results of the experiment underway in the Markham Stouffville area will help provide some pieces of the puzzle in how to develop an integrated health system that truly functions in the interests of its community. MSH is hopeful that the findings will be transferable to other communities. However, we realize that some of MSH's community, institutional and cultural characteristics may limit applicability elsewhere. For example, the MSH community is growing, resources are limited and competition among 
healthcare agencies is relatively minor.

Of equal importance is the hospital's willingness to use its capital reserves to seed the process of change. These attributes may not be universally present in other communities.

Incentives must ultimately address the basic issue of dollars and cents. The Health Services Restructuring Commission investigated the barriers to implementing integrated health systems in Ontario (HSRC 1999). The HSRC concludes that although much can be done with "goodwill" fundamental change will require legislative levers. In fact, there is no legal basis for an IHS to exist.

Many communities, including Markham Stouffville, have decided that it is better to move ahead a few steps than have the opportunities for change and leadership pass by. The actions and strategies being pursued can be effective. They can improve care and consumer satisfaction. They can help utilize increasingly scarce resources more effectively. An IHS offers a model of delivery that can move us from myth to reality. It provides improved accessibility, a populationhealth-based focus, cost-effective solutions and a better coordinated continuum of care.

In recent months, Minister's speeches have begun to use the language of integrated systems again. The Premier and others are promoting the concept of primary care. Movement towards integrated health systems may slowly be starting to take root. As was once said, "Considering the direction things are going, it's impossible to predict in which direction they'll be going next" (Brilliant 1994).

\section{References}

Avrich, Cheryl et. al. 1997. "Southeast York Region Integrated Health System: Creating Canada's Healthiest Community."

Brilliant, Ashleigh. 1994. I Feel Much Better Now That I've Given Up Hope. Santa Barbara: Woodbridge Press.

Goldsmith, Jeff, 1998. "Reconsidering Integration Strategies." Healthcare Financial Management. (April).

Hospital Management Research Unit (HMRU, University of Toronto). 1996. "Integrated Delivery Systems - Providing a Continuum of Health Care.” Working paper. Technical Report No. 96-02.

Ontario College of Family Physicians (OCFP). 1995. "Bringing the Pieces Together: Planning for Future Health Care.” Toronto.

Ontario College of Family Physicians (OCFP). 1999. "Family Medicine in the 21st Century: A Prescription for Excellence in Health Care." Available at www.cfpc.ca/ocfp/commun/ fp2000.html.

Ontario Health Services Restructuring Commission. 1997. "GTA/905 Health Services Restructuring Report.” Toronto.

Ontario Health Services Restructuring Commission. 1999. "Implementing Integrated Health Systems in Ontario: A Review of Legislative/Regulatory Implications.” Toronto.

Quigley, Maureen A. 1996. "Integrated Health Systems: Report of Focus Groups On Ministry of Health Policy Framework." Ontario Ministry of Health, Toronto.

Rosser, Walter. W. and Jan Kasperski. 1999. "Organizing Primary Care for an Integrated System." HealthcarePapers. (Winter) 1(1): 5-21.

Toronto District Health Council (TDHC). 1996. "Creating Integrated Health Delivery Systems for Metropolitan Toronto." Toronto

Toronto District Health Council (TDHC). 1999. "Enhancing the Delivery of Primary Care in Toronto: A Conference for Family and General Practitioners." Toronto

York Region District Health Council. 1995. "York Region Acute Care Study.” Newmarket 